\title{
Stress sensitivity evaluation of ultra-low permeability sandstone reservoir and its influence on oilfield development
}

\author{
Xin Bai ${ }^{1}$, Chunfen Guo*1, Xingli Yang ${ }^{1}, \mathrm{Xi} \mathrm{Liu}^{1}$ \\ ${ }^{1}$ Yanchang Oilfield Limited Company, Yan'an Shaanxi 716000, China
}

\begin{abstract}
Luohe ultra-low permeability sandstone reservoir is a hot block in Yanchang oilfield, which is a potential point for increasing production and reservoir. In view of the current situation that there is no unified stress sensitivity evaluation standard for ultra-low permeability sandstone in the study area, taking the ultralow permeability sandstone in Luohe district as the research object, the stress sensitivity evaluation of ultralow permeability sandstone is carried out by using experimental analysis as the main means. The results show that it is more accurate to evaluate porosity by using pore stress sensitivity coefficient instead of pore compressibility coefficient. With the increase of net overburden pressure, the porosity stress sensitivity decreases gradually; the permeability stress sensitivity is evaluated by variable confining pressure. With the increase of confining pressure, the permeability damage decreases. With the decrease of confining pressure, the permeability damage increases, but it can not recover to the original value, so the permeability damage is irreversible; in the low bottom hole pressure stage, stress sensitivity has a greater impact on oil well productivity, while in the high bottom hole pressure stage, stress sensitivity has a smaller impact on oil well productivity; advanced water injection can reduce the adverse effect of stress sensitivity on the development of ultra-low permeability sandstone and maximize the economic benefits. The research results clarify the method of stress sensitivity evaluation, and provide guidance for efficient water injection in the next step.
\end{abstract}

\section{Introduction}

With the increasing degree of oil production, crude oil is continuously produced, which will reduce the formation pore pressure, change the stress state of surrounding rock and affect the physical properties of reservoir. The change of porosity and permeability is directly related to the productivity of oil wells[1]. The relevant data show that the local formation pressure decreases by $5 \%$, and the production decreases by about $13 \%$. Therefore, it is very important to carry out reservoir stress sensitivity evaluation. Reservoir stress sensitivity evaluation includes porosity sensitivity evaluation and permeability sensitivity evaluation[2-3]. For ultra-low permeability reservoirs, drilling factors and oil production factors will lead to stress sensitivity. With the increase of overlying net pressure, porosity decreases continuously, which has been generally recognized. However, there are different opinions on the evaluation criteria, such as pore volume compressibility method, effective stress method, there is no unified evaluation standard. There are great differences between low permeability reservoir and high permeability reservoir, which have the characteristics of small pores, complex structure and large specific surface area[4]. It is of great significance to carry out the corresponding stress sensitivity evaluation according to the characteristics of ultra-low permeability reservoir.
Luohe ultra-low permeability sandstone has the characteristics of high reservoir pressure coefficient, low single well productivity, obvious fracturing effect and no obvious water injection effect. Advanced water injection is a common way of water injection in ultra-low permeability reservoir, which can maintain high formation pressure and reduce permeability damage caused by formation pressure drop. The selection of advanced water injection timing is very important, and the daily oil production is different at different water injection timing[5]. Through stress sensitivity evaluation, the water injection time can be determined to maximize the economic benefits of advanced water injection. Through the stress sensitivity evaluation of ultra-low permeability sandstone, the damage changes of porosity and permeability can be identified, which provides a reference for the next step of water injection timing selection, and then provides ideas for improving the productivity of ultra-low permeability sandstone reservoir.

\section{2 methods and materials}

\section{1 materials and instruments}

DXD high precision digital pressure sensor (Zhejiang Yangzijiang Pump Industry Co., Ltd.), CMS-300 high temperature and high pressure porosimeter (Hangzhou mico Sensing Technology Co., Ltd.), mf1035c electronic

\footnotetext{
*Corresponding author: mma0405@sina.com
} 
analytical balance (Shandong bock Instrument Technology Co., Ltd.), gilibrato soap flow meter (American sensedyne company).

Experimental materials: the core is from Yanchang Formation of Luohe oilfield, and the core sampling is shown in Table 1.

Table1. list of core sampling

\begin{tabular}{|c|c|c|c|c|}
\hline number & $\begin{array}{c}\text { Well } \\
\text { location }\end{array}$ & $\begin{array}{c}\text { Well } \\
\text { section } \\
(\mathbf{m})\end{array}$ & $\begin{array}{c}\text { porosity } \\
\text { (\%) }\end{array}$ & $\begin{array}{c}\text { permeability } \\
\text { (mD) }\end{array}$ \\
\hline 1 & H78 & 1807.7 & 9.08 & 0.94 \\
\hline 2 & H52 & 1984.08 & 8.88 & 0.77 \\
\hline 3 & H58 & 1804.68 & 10.32 & 0.96 \\
\hline 4 & H90 & 1927.90 & 11.24 & 0.54 \\
\hline 5 & H43 & 2038.54 & 10.15 & 0.34 \\
\hline 6 & H50 & 2004.76 & 8.7 & 0.22 \\
\hline 7 & H53 & 1989.96 & 9.91 & 0.52 \\
\hline 8 & H87 & 1931.63 & 9.09 & 0.41 \\
\hline 9 & H30 & 1982.49 & 8.4 & 0.64 \\
\hline 10 & H21 & 2036.95 & 9.32 & 0.92 \\
\hline
\end{tabular}

\section{2 experimental methods}

The porosity stress sensitivity is evaluated by the porosity stress sensitivity coefficient, and the formula is as follows:

$$
C_{p}=-\frac{1}{v_{p}} \frac{d v_{p}}{d \delta}
$$

Where: CP: pore stress sensitivity coefficient, dimensionless; $\delta$ : Overlying stress increment, MPa; VP: pore volume, $\mathrm{cm} 3$.

The permeability sensitivity evaluation is obtained by variable confining pressure experiment. The confining pressure of the core is gradually increased, and the permeability damage coefficient is recorded; for the core, the confining pressure is gradually reduced, and the permeability damage recovery coefficient is recorded, so as to determine the permeability stress sensitivity evaluation.

\section{Results and analysis}

\section{1 porosity stress sensitivity evaluation}

Reservoir stress sensitivity evaluation includes porosity stress sensitivity evaluation and permeability stress sensitivity evaluation. Porosity stress sensitivity evaluation is usually based on overlying net stress. This method ignores the change of pore compressibility and is difficult to truly reflect the change of porosity stress sensitivity. By fitting the pore stress sensitivity coefficient and the pore stress sensitivity, it is found that there is a strong correlation between them. When the pore stress sensitivity coefficient increases, the pore stress sensitivity also increases. Taking the pore stress sensitivity coefficient as a parameter can solve this problem well.

It can be seen from Figure 1 that when the porosity is less than $9.0 \%$, the stress sensitivity of porosity is strong, but when the porosity is more than $9.0 \%$, the stress sensitivity of porosity is weak. Therefore, the stress sensitivity of low porosity reservoir composed of intergranular pores is stronger than that of high porosity reservoir composed of dissolution pores

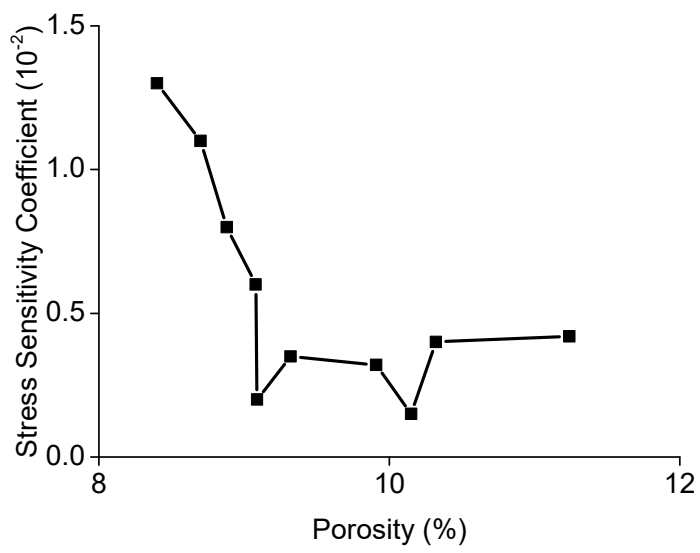

Figure 1. Pore stress sensitivity coefficient and porosity scatter diagram (this diagram needs to be modified according to the data in the table above)

\section{2 permeability stress sensitivity evaluation}

\subsection{1 sensitivity of permeability damage coefficient}

The permeability stress sensitivity is obtained by the variable surrounding rock experiment. It can be seen from Fig. 2 that the permeability damage coefficient is $19.75 \%$ under the pressure of $2 \mathrm{MPa}$; when the pressure is $4 \mathrm{MPa}$, the permeability damage coefficient is $14.5 \%$; when the pressure is $6 \mathrm{Mpa}$, the permeability damage coefficient is $12.3 \%$; the pressure coefficient is $8 \mathrm{MPa}$ and the permeability damage coefficient is $11.3 \%$; the critical pressure is the inflection point of permeability damage coefficient near the pressure of $5 \mathrm{MPa}$. 

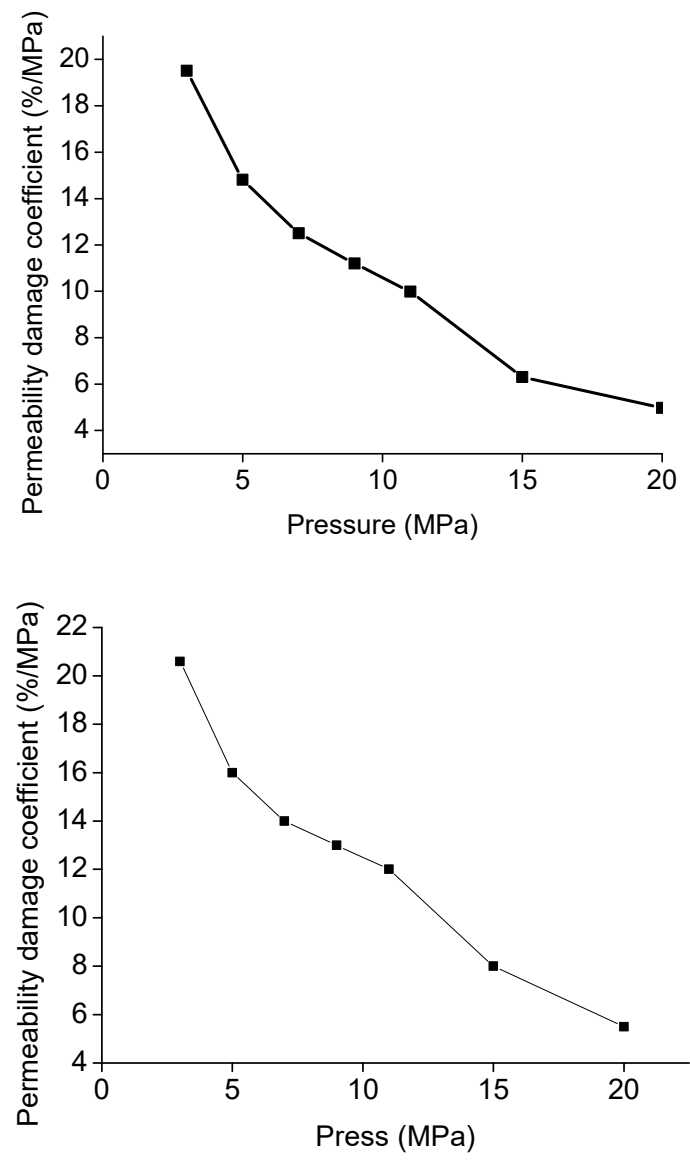

Figure 2. Permeability damage coefficient curves of different samples (a, No. 1; b. 2)

\subsection{2 sensitivity of permeability damage rate}

According to industry regulations, permeability damage rate $(\mathrm{DK} *)$ is usually divided into six grades, and $\mathrm{DK} * \leq$ 5 is no damage; $5<\mathrm{DK} * \leq 30$ is weak damage; if $30<$ $\mathrm{DK} * \leq 50$, it is medium weak; if $50<\mathrm{DK} * \leq 70$, it is medium to strong; $70<\mathrm{DK} * \leq 90$, it is strong damage; $90<\mathrm{DK}^{*}$, it is very strong damage. It can be seen from table 2 that the permeability damage coefficient of Yanchang formation is concentrated in 80-90 in the process of pressure rise, and 40-50 in the process of pressure drop. Generally, the pressure drop process is used as the evaluation index, so it can be seen that the stress sensitivity is medium to weak.

Table2. Results of stress sensitivity assessment in the study area

\begin{tabular}{|c|c|c|}
\hline Sample number & Boosting process & $\begin{array}{c}\text { Depressurization } \\
\text { process }\end{array}$ \\
\hline 1 & 84 & 45.2 \\
\hline 2 & 92.1 & 46.9 \\
\hline 3 & 81.5 & 56.9 \\
\hline 4 & 87.8 & 45.7 \\
\hline
\end{tabular}

The results show that the clay minerals directly affect the reservoir sensitivity. The clay minerals in the study area are mainly illite and kaolinite $(45.8 \%)$, followed by illite and kaolinite. When the fluid velocity increases to a certain extent, it is easy to fall off when it meets water in the soft place, and then block the pores and damage the physical properties of the reservoir.

\section{3 capacity evaluation}

Low permeability glutenite is a low saturation reservoir with insufficient natural energy, mainly elastic drive. NPR (pressure drop value of $1 \%$ of the geological reserves) $=$ $0.0023<2$, DPR (ratio of oil production to closed elastic driving energy) $=5.758>2.5 \mathrm{MPa}$. According to the classification standard of natural energy driving, it belongs to the range of natural energy deficiency.

In order to study the effect of stress sensitivity on productivity, two aspects of research are carried out. On the one hand, the productivity changes under the same pressure sensitivity coefficient. It can be seen from Fig. $3 \mathrm{~A}$ that in the stage of low bottom hole pressure, stress sensitivity has a greater impact on the productivity of oil wells; in the stage of high bottom hole pressure, stress sensitivity has little effect on oil well productivity. In addition, we can see from Fig. 3B that with the increase of the stress sensitivity coefficient, the productivity decreases and the reduction range increases. Therefore, when stress sensitivity is considered, the permeability reservoir should be developed under lower pressure difference.
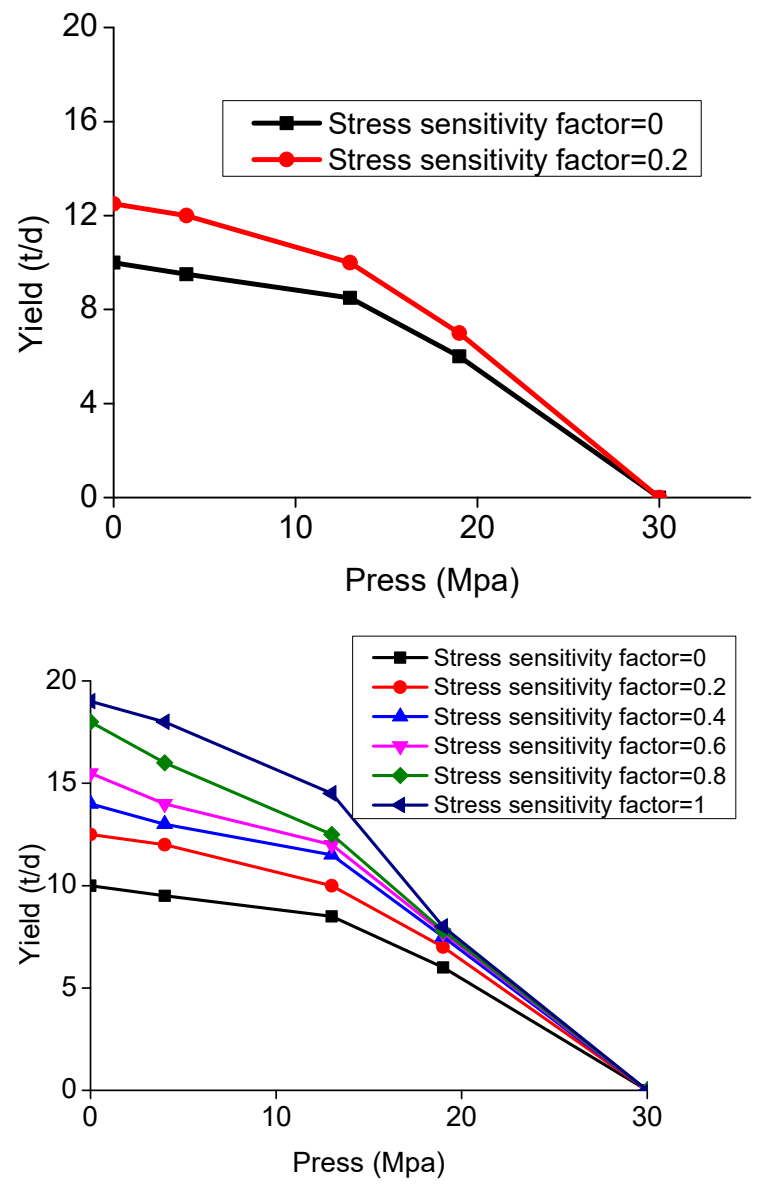

Figure 3. Stress sensitivity coefficient and productivity curve (a, the same stress sensitivity coefficient; b, Different stress sensitivity coefficient) 


\section{4 timing of water injection development in ultra-low permeability reservoir}

Stress sensitivity effect is one of the important factors affecting the development effect of ultra-low permeability reservoir. According to the above permeability stress sensitivity experiment, the permeability decreases with the increase of confining pressure; with the decrease of confining pressure, the permeability begins to recover slowly and increases continuously, but it can not recover to the original value, and the permeability can only recover to about the original value. Therefore, early water injection with formation pressure can reduce reservoir permeability damage caused by pressure drop. Advanced water injection is a technology for ultra-low permeability reservoir with the characteristics of elastic-plastic deformation, which can reduce the permeability damage caused by the decrease of formation pressure. Stress sensitivity is closely related to the timing of water injection. Because of the existence of stress sensitivity, the development mode of advanced water injection should be selected for ultra-low permeability reservoir.

Reasonable water injection time refers to the time of maximizing economic benefits, when the cost is the lowest and the recovery is the highest. The earlier the water injection time, the higher the cost, but the higher the recovery. According to the relationship between water injection time and NPV, it can be concluded that with 0 as the boundary, $<0$ represents advanced water injection, and $>0$ represents delayed water injection. With the change of water injection time, the NPV of advanced water injection stage is increasing, while the NPV of delayed water injection stage is decreasing. Therefore, for ultra-low permeability reservoir, advanced water injection can maximize economic benefits. The monthly oil production of early water injection ranged from $45.2 t$ to $70.66 \mathrm{t}$, with a monthly average of $55.02 \mathrm{t}$. The monthly oil production of water injection in the same period ranged from $21.1 \mathrm{t}$ to $69.5 \mathrm{t}$, with a monthly average of $40.21 \mathrm{t}$. The monthly oil production of delayed water injection ranged from $31.1 \mathrm{t}$ to $68.2 \mathrm{t}$, with a monthly average of $34.79 \mathrm{t}$; the monthly oil production of early water injection is obviously higher than that of the other two water injection opportunities, so it has economic benefits to advance water injection for low permeability reservoirs in the study area.

\section{5 case analysis}

Four wells in the study area are selected to analyze the water injection timing on the basis of reservoir stress sensitivity evaluation. Among them, well X1 is non stress sensitive, the other three wells are stress sensitive, $\mathrm{X} 2$ is advanced water injection, $\mathrm{X} 3$ is simultaneous water injection, $\mathrm{X} 4$ is delayed water injection. It can be found that the monthly oil production of well X1 is $40.82 \mathrm{t} \sim$ $34.22 \mathrm{t}$, with a monthly average of $38.79 \mathrm{t}$; the monthly oil production of well X2 is $38.35 \mathrm{t} \sim 20.67 \mathrm{t}$, with a monthly average of 28.64t; the monthly oil production of well X3 is $21.65 t \sim 17.65 t$, with a monthly average of $22.35 t$; the monthly oil production of well X4 ranges from $20.98 \mathrm{t}$ to 13.25 t, with a monthly average of $19.58 \mathrm{t}$. Compared with
$\mathrm{X} 1$ and $\mathrm{X} 2$, the monthly oil production of $\mathrm{X} 1$ is higher than that of $X 2$, which indicates that the existence of stress sensitivity will slow down the formation pressure and reduce the monthly oil production rate. Compared with $\mathrm{X} 2, \mathrm{X} 3$ and $\mathrm{x} 4$, the monthly oil production of $\mathrm{X} 2$ is higher than that of X3 and X4. Advance water injection can improve the monthly oil production and slow down the decline rate of formation pressure.

\section{4 conclusion}

(1) Reservoir sensitivity evaluation includes pore stress sensitivity evaluation and permeability stress sensitivity evaluation. The pore stress sensitivity coefficient is used as an index to evaluate the pore stress sensitivity. The pore pressure sensitivity coefficient is based on the residual pore compression coefficient, which has higher accuracy; using the variable confining pressure experiment to analyze the sensitivity of permeability, it is concluded that with the increase of confining pressure, the permeability decreases, the confining pressure decreases, and the permeability gradually recovers, but it can not recover to the original value, so the permeability loss is irreversible.

(2) The effect of stress sensitivity on productivity is reflected in the stage of low bottom hole pressure, and the effect of stress sensitivity on productivity is greater than that in the stage of high bottom hole pressure; therefore, when stress sensitivity is considered, ultra-low permeability reservoir can be developed under low pressure difference. However, when the bottom hole pressure drops by $5 \mathrm{MPa}$, the production of the two areas decreases by about $20 \%$. Through the analysis of different stress sensitivity coefficients, the production of the oil well decreases by about $10 \%$ when the stress sensitivity coefficient increases by 0.02 ; according to the principle of conservation of reservoir material, it is considered that adopting advanced water injection in Yanchang oilfield can reduce the adverse effect of stress sensitivity on the development of low permeability glutenite reservoir.

\section{References}

1. Hou Z K, Cheng H L, Sun S W, et al. Crack propagation and hydraulic fracturing in different lithologies[J]. Applied Geophysics, 2019, 16(2):243251.

2. Li W, Ma F, Zhao G. Composition and dynamics of sulfate-reducing bacteria during the waterflooding process in the oil field application[J]. Bioresource Technology, 2010, 101(8):2643-2650.

3. Yu J, Pan B Z, Yang Q S. Characteristics of resistivity $\log$ response of oil layers under polymer flooding[J]. Applied Geophysics, 2012, 9(2):187-194.

4. Khosravi J , Alamdari A. Copper removal from oilfield brine by coprecipitation[J]. Journal of hazardous materials, 2009, 166(2-3):695-700.

5. Malik S, Zhang Y M, Asimi M A, Gould T L. Steamflood with vertical injectors and horizontal producers in multiple zones[J]. Spe Reservoir Evaluation \& Engineering, 2011, 14(2):161-170. 